\title{
Metal Ion Implantation for the Fabrication of Stretchable Electrodes on Elastomers
}

\author{
By Samuel Rosset, * Muhamed Niklaus, Philippe Dubois, and Herbert R. Shea
}

Here, the use of low-energy metal ion implantation by filtered cathodic vacuum arc to create highly deformable electrodes on polydimethylsiloxane (PDMS) membranes is reported. Implantation leads to the creation of nanometer-size clusters in the first $50 \mathrm{~nm}$ below the surface. When the elastomer is stretched, these small clusters can move relative to one another, maintaining electrical conduction at strains of up to $175 \%$. Sheet resistance versus ion dose, resistance versus strain, time stability of the resistance, and the impact of implantation on the elastomer's Young's modulus are investigated for gold, palladium, and titanium implantations. Of the three tested metals, gold has the best performance, combining low and stable surface resistance, very high strain capabilities before loss of electrical conduction, and low impact on the Young's modulus of the PDMS membrane. These electrodes are cyclically strained to $30 \%$ for more than $10^{5}$ cycles and remain conductive. In contrast, sputtered or evaporate metals films cease to conduct at strains of order $3 \%$. Additionally, metal ion implantation allows for creating semi-transparent electrodes. The optical transmission through 25- $\mu \mathrm{m}$-thick PDMS membranes decreases from $90 \%$ to $60 \%$ for Pd implantations at doses used to make stretchable electrodes. The implantation technique presented here allows the rapid production of reliable stretchable electrodes for a number of applications, including dielectric elastomer actuators and foldable or rollable electronics.

single deformation of a few percent (for example for an implantable sensor that needs to adapt its shape to the environment in which it is inserted), to repeatable stretching above $100 \%$ strain in the case of DEAs. While not the largest market, DEAs are the most demanding application for stretchable electrodes, combining large strains and large number of cycles. In this paper, we focus on electroactive polymer applications as an illustration of the use of these electrodes.

Electrodes for macroscale DEAs are generally made with carbon powders, ${ }^{[5,6]}$ carbon greases, ${ }^{[5]}$ or carbon powders mixed with unpolymerized elastomer. ${ }^{[7]}$ Although they can be easily and rapidly applied on a $\mathrm{cm}^{2}$ to $\mathrm{mm}^{2}$ scale, carbon-based electrodes are not applicable to the miniaturization of DEAs for which patternable, clean, and reliable compliant electrodes are needed. Metal thin-film deposition is not an option, because metal films can only sustain a few percent of deformation before breaking, and their high Young's modulus (4 to 5 orders of magnitude higher than that of the elastomers typically used to make DEAs) stiffens

\section{Introduction}

The emergence of the use of polymers as functional materials, as well as the fabrication of electronic circuits on flexible and deformable substrates, has brought the need for electrodes that can be stretched, bent or deformed while remaining conductive. Dielectric elastomer actuators (DEAs, also known as artificial muscles), ${ }^{[1]}$ expandable and rollable displays ${ }^{[2,3]}$ and flexible electronics ${ }^{[4]}$ are three examples of applications that require important deformation or stretching of a conducting material. The amount of strain that the electrode must be able to sustain without damage, as well as the number of strain cycles to which the electrode will be submitted, depends on the application. It can vary from a

[*] S. Rosset, M. Niklaus, Dr. P. Dubois, Prof. H. R. Shea Microsystems for Space Technologies Laboratory Ecole Polytechnique Fédérale de Lausanne Rue Jaquet-Droz 1, cp 526, 2002 Neuchâtel (Switzerland) E-mail: samuel.rosset@a3.epfl.ch

DOI: $10.1002 / \mathrm{adfm} .200801218$ the structures and drastically reduces the actuators' performance. Several ingenious electrode designs have been published that made use of metallic thin-film to create deformable electrodes, such as in-plane s-shaped ${ }^{[8]}$ or z-shaped ${ }^{[9]}$ strips, or out-of-plane buckled wavy electrodes. ${ }^{[10]}$ However, the in-plane patterned electrodes are not well adapted to the typical biaxial strain produced by DEAs, and the buckled electrodes require the elastomer to be stretched during the metal deposition, which is not compatible with microfabrication. Pimpin et al. successfully applied thin-film electrodes on small-size DEAs by patterning the metal in concentric rings, ${ }^{[11]}$ but the fabrication of a $\varnothing 2 \mathrm{~mm}$ actuator requires patterning shapes with some dimensions lower than $10 \mu \mathrm{m}$, which can be problematic in making smaller devices.

Several research groups have tried to avoid the shortcomings of plain metallic thin films, for example by using nanoporous gold films capable of $25 \%$ strains, ${ }^{[12]}$ or fabricating deformable electrodes based on precipitation and diffusion of platinum nanometer-sized clusters from a Pt salt. ${ }^{[13]}$ Electrodes created with this technique are intrinsically photopatternable, can be stretched up to $150 \%$ before loss of electrical conduction and can survive 1000 stretching cycles to $30 \%$ strain. 
We have introduced low-energy metal ion implantation by filtered cathodic vacuum arc (FCVA) as a means to create compliant electrodes for DEAs. They present a low surface resistance $(<1 \mathrm{k} \Omega$ per square) that does not drift with time, they can be stretched up to $175 \%$ strain and survive more than 100000 cyclic deformations to $30 \%$ strain, and they have a low-tomoderate impact on the Young's modulus of the elastomer on which they are created $(+50 \%$ relative increase). This technology enabled us to fabricate small-size diaphragm DEAs capable of out-of-plane displacements up to $7 \%$ of their diameter, ${ }^{[14,15]}$ which was increased to $25 \%$ of the membrane's diameter after optimization. This paper reports on the mechanical and electrical characteristics of titanium, gold, and palladium implanted electrodes on polydimethylsiloxane (PDMS) thin $(20-30-\mu \mathrm{m}$ thick) layers and membranes.

\section{Metal Ion Implantation}

The key point of ion implanted electrodes resides in the fact that implantation does not form a continuous polycrystalline film in which grains are attached to each other at the grain boundaries, thus forming a rigid film capable of only a few percent strain before breaking. Instead, ion implantation leads to the formation of small size $(2-20 \mathrm{~nm})$ clusters in the polymer matrix (Fig. 1). These clusters can touch each other (thus providing a conducting path) without forming a strong mechanical bond. The absence of a strong bond between clusters allows them to slide relative to each other (Fig. 2). This leads to a reduced impact on the stiffening (increase of Young's modulus) of the PDMS due to the inclusion of the metallic particles, and an ability to withstand high strains before losing electrical conduction.

Creating a conductive film by implantation of metallic particles at the surface of a PDMS layer requires low acceleration energy $(<10 \mathrm{keV})$ to keep the ions close to the surface $(<50 \mathrm{~nm})$, and a high ion flux in order to reach the desired film resistivity after a reasonable amount of time. Conventional beamline implanters cannot usually deliver high ion flux at low energies, and can therefore not be used. We use a plasma-based implantation

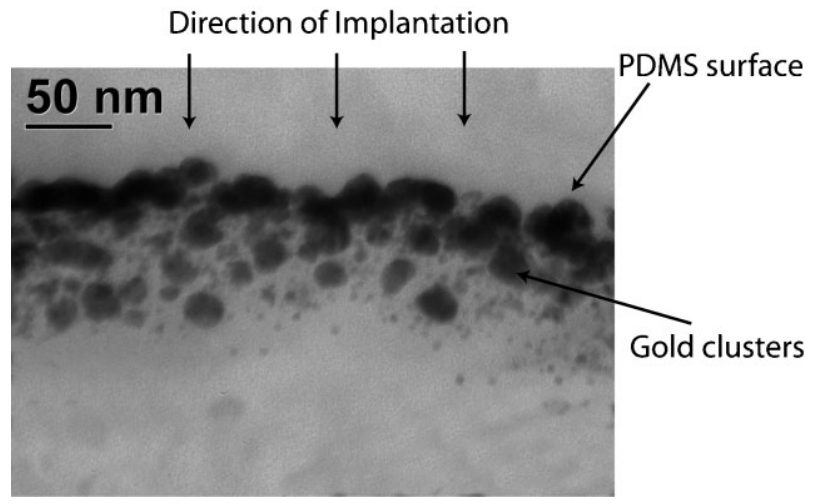

Figure 1. Transmission electron microscopy (TEM) cross-section of Auimplanted PDMS (dose of $1.5 \times 10^{16}$ at cm $^{-2}$ at $5 \mathrm{keV}$ ), which shows the Au atoms forming $\mathrm{nm}$-size clusters when implanted into PDMS [16]. The atoms extend from the surface down to a depth of about $60 \mathrm{~nm}$ below the surface.

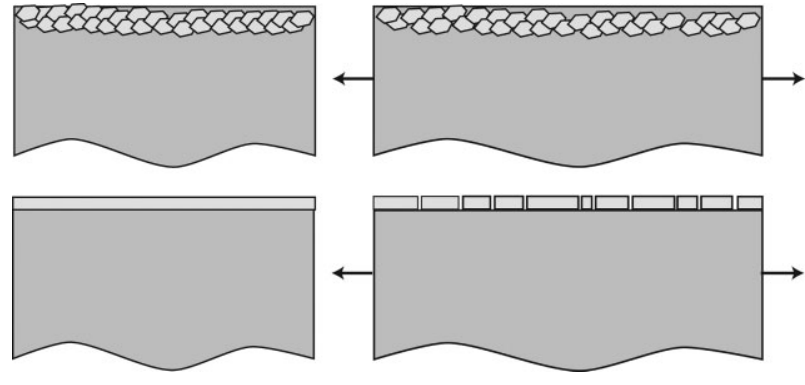

Figure 2. Top: schematic cross-section illustrating a compliant electrode obtained by ion implantation. The clusters are touching each other but can slide relative to each other. They can sustain large strain while remaining conductive. Bottom: non-compliant electrode obtained by thin-film deposition. The strong binding forces which link the metallic atoms together drastically limit the achievable strain before damage to the electrode.

technique called filtered cathodic vacuum arc, ${ }^{[17]}$ which allows us to obtain high ion flux at low energies $(0.2-10 \mathrm{keV})$ : for gold implantation, 3 minutes are sufficient to obtain a surface resistance below $1 \mathrm{k} \Omega$ per square on our system. Our experimental FCVA implanter has a beam size of $\sim 1 \mathrm{~cm}^{2}$ and works in pulsed mode. The implanted dose depends linearly on the number of arc pulses. Rutherford backscattering spectroscopy (RBS) calibrations were made to correlate the number of pulses with the actual implanted dose $\left(\right.$ at $\left.^{-2}\right)$. The acceleration voltage decreases during each arc pulse, which leads to an energy distribution of the incoming ions between $0.4-5 \mathrm{keV}$, thus ensuring a penetration distribution of the implanted species between $50 \mathrm{~nm}$ and the surface of the elastomer.

High resolution patterning of the ion implantation can be achieved by the use of selective masking, which will protect defined zones that will remain free of ions. This selective masking was performed by laser-milled steel shadow masks that are applied on the PDMS layer during the implantation. If very small structures need to be made $(<100 \mu \mathrm{m})$, a photolithography step can be used to pattern a mask on the PDMS surface on which the implantation will be conducted, similar to a lift-off process.

\section{Resistance and Time Stability}

The first step to test the applicability of FCVA ion implantation to create compliant electrodes for DEAs consists of measuring the surface resistance of implanted PDMS, as well as its stability in time, in order to show this technique's ability to create stable conductive layers.

Chips for resistance measurements were prepared by spincoating a $20-\mu \mathrm{m}$-thick PDMS layer onto a 4 inch silicon wafer. The coated wafer was then diced into $10 \times 10 \mathrm{~mm}^{2}$ chips, and gold contact electrodes ( $\sim 25$-nm thick) were sputtered on the PDMS. The spacing between the two electrodes was $0.5 \mathrm{~mm}$ wide on a length of $5 \mathrm{~mm}$, thus defining a zone of $1 / 10$ square. Implantation was then conducted through a steel shadow mask that defined an opening covering the two electrodes, as well as the space between them. The implanted layer's resistance was measured with a multimeter between the two gold pads. For $\mathrm{Ti}, \mathrm{Au}$, and $\mathrm{Pd}$, different doses were used, starting at 
$9 \times 10^{15}$ at $\mathrm{cm}^{-2}$. The surface resistance of each sample was measured with a multimeter immediately after implantation. Additionally, the resistance was regularly monitored to record the time evolution of the implanted layers' electrical properties.

Transmission electron microscopy (TEM) images of implanted PDMS show that when the metallic ions penetrate the polymer's surface, they tend to form clusters whose size depends on the dose. $^{[16]}$ At low doses $\left(<10^{16}\right.$ at $\mathrm{cm}^{-2}$ for $\left.\mathrm{Au}\right)$ the implanted particles' density is low and they are too far apart from each other to create a conductive path. Consequently, resistance remains very high (above $200 \mathrm{M} \Omega$ ). For higher doses, a sharp decrease in resistivity is observed. This is the percolation threshold: the density of metallic particles becomes high enough that conduction paths appear in the implanted layer. For doses higher than the percolation threshold, the implanted layer's properties tends toward those of a thin-film metallic layer. For $\mathrm{Au}$, the region between the percolation and low-resistance regions is particularly narrow: the surface resistivity drops from $>10^{9} \Omega$ per square to $1 \mathrm{k} \Omega$ per square for doses between $10^{16}$ at $\mathrm{cm}^{-2}$ and $1.5 \times 10^{16}$ at $\mathrm{cm}^{-2}$ (Fig. 3). For titanium, the percolation begins for the same dose, but is much less sharp, and the surface resistance at higher doses is larger than for gold. Palladium has a percolation threshold below $1 \times 10^{16}$ at $\mathrm{cm}^{-2}$, which is therefore not visible on this set of samples. The percolation threshold of Pd-implanted layers occurring at low doses, combined with the high ion flux obtained with Pd on our FCVA system, makes it difficult to obtain layers in the percolation zone. Pd-implanted layers already have an interesting surface resistance for doses around $1 \times 10^{16}$ at $\mathrm{cm}^{-2}$, and obtaining a low surface resistance with less metallic particles has a very positive influence on the conduction/ stiffening tradeoff that will be described in details later.

Time stability of the resistivity is a concern for titanium layers, which suffer from oxidation: their resistivity quickly increases if left in ambient air, unless they are heavily implanted. On the other hand, gold-implanted layers exhibit very stable resistivity versus time for doses higher than $1.2 \times 10^{16}$ at $\mathrm{cm}^{-2}$. Below this limit, the resistivity increases with time, probably due to diffusion. The same behavior is observed for Pd implantations (Fig. 4); for both

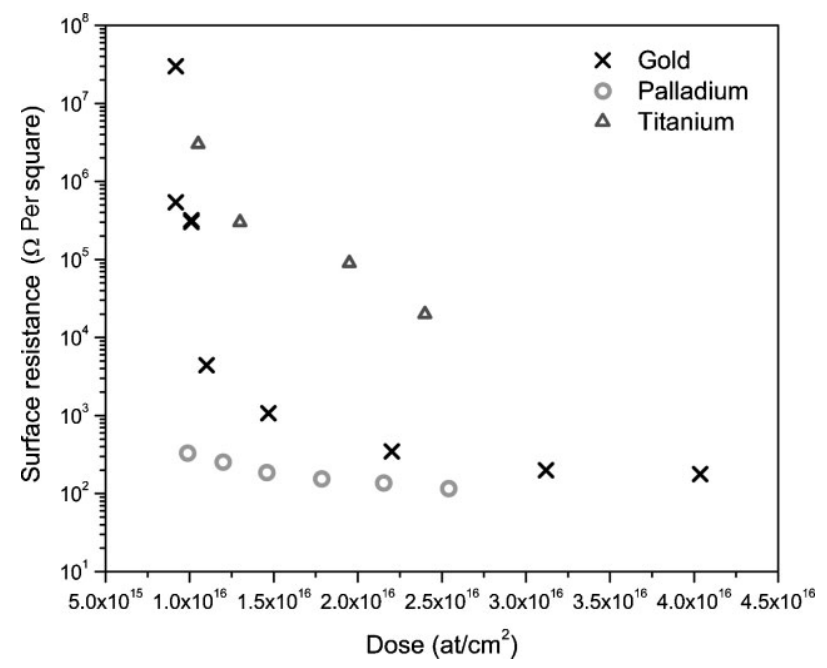

Figure 3. Surface resistance versus dose obtained for $\mathrm{Au}, \mathrm{Pd}$, and $\mathrm{Ti}$ implantations at $5 \mathrm{keV}$. The percolation threshold is clearly visible for gold.
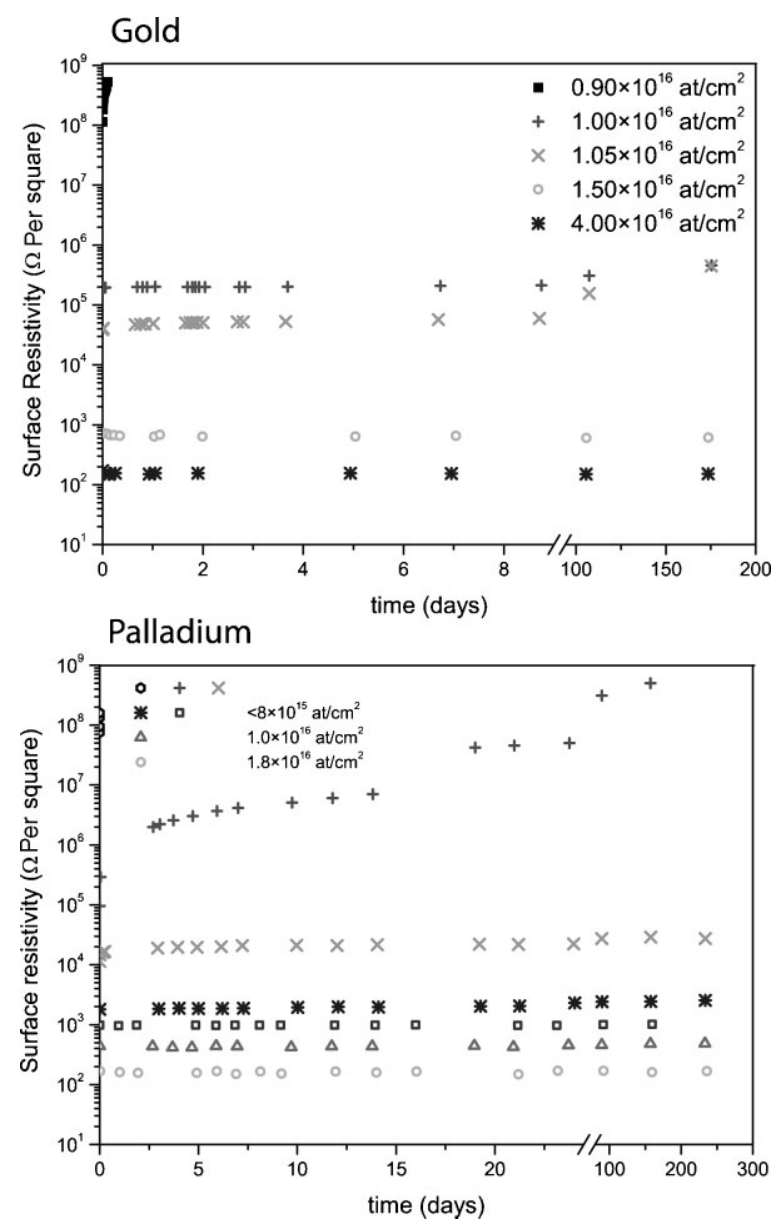

Figure 4. Time stability of the surface resistivity of $\mathrm{Au}$ - (top) and Pdimplanted (bottom) PDMS for different metal doses. Above a critical ion dose, the surface resistance is highly stable and was not observed to evolve over a period of more than 8 months.

metals, implanted layers with an initial resistivity below $10 \mathrm{k} \Omega$ per square exhibit an excellent time stability over more than 8 months, and layers with an initial sheet resistance above $10^{8} \Omega$ per square become non-conductive in a matter of minutes. Because Ti layers have higher resistances compared to the two noble metals, coupled with oxidation and degradation problems, titanium implantations were not used for strain measurements.

\section{Maximum Strain Capabilities}

Having shown in the previous section that ion implantation allows for the creation of conductive layers that are stable in time, the strain capabilities of these electrodes were investigated: i) How does the resistance change when the implanted layer is deformed? ii) What is the maximal achievable strain at which the layer ceases to be conductive? iii) Is there a degradation of the resistance for cyclic deformation? These are the three questions addressed in this section.

Uniaxial tensile strain was applied on implanted PDMS strips using a linear stage connected to a stepper motor. The samples 


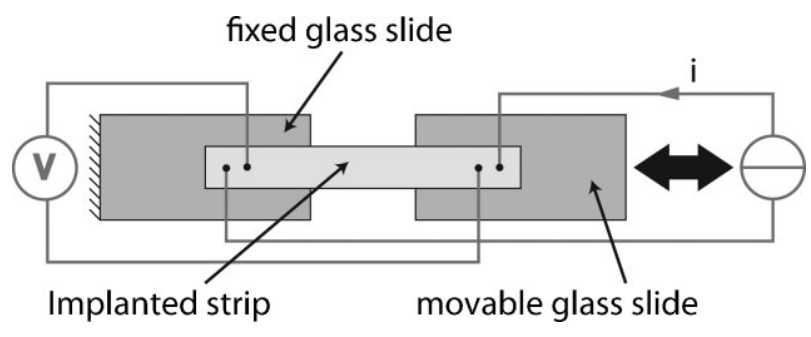

Figure 5. Samples for strain tests: an implanted PDMS strip's extremities are glued to a microscope glass slide. Wires are then connected to the stripe for a four-point resistance measurement.

consist of $\sim 200-\mu \mathrm{m}$-thick PDMS strips of a length of $6-20 \mathrm{~mm}$ and a width of $3-5 \mathrm{~mm}$, which were implanted over the whole area on one side. Each extremity of the strip was then glued to a glass slide and electrical connections were added (Fig. 5). This configuration ensured the electrical contacts remained free of mechanical stress and strain: any change in resistance while stretching the sample was therefore attributed to the implanted layer. Two wires were connected on each side of the strip, and 4wire resistance measurements were performed in order to suppress any possible influence of the contacts.

The influence of dose was studied by implanting PDMS strips with different doses of gold ions at $5 \mathrm{keV}$, and stretching them while recording their resistance. Sputtered samples $\left(t_{\mathrm{Au}} \approx 25 \mathrm{~nm}\right)$ were also fabricated to compare metal ion implantation's elongation capabilities with those of plain metallic electrodes. The maximal strain before loss of conductivity $\left(s_{\max }\right)$ is found to be very dependent on the dose and deposition method (Fig. 6). Because the samples were larger than the ion beam size, a motorized scanning stage was used to move the sample under the beam during the implantation. The RBS calibration is not valid in this case, and doses are consequently given in arc pulses per surface area throughout this section.

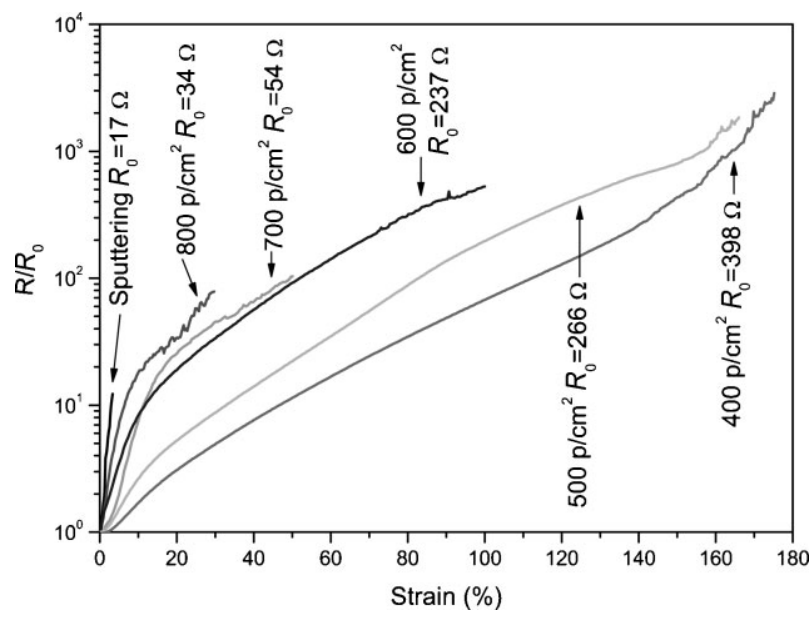

Figure 6. Change in resistance $\left(R / R_{0}\right.$, where $R_{0}$ is the resistance at zero strain) of gold-sputtered and gold-implanted PDMS strips under uniaxial strain, up to loss of conductivity. The implanted dose are given in pulses per square centimeter, due to the difficulty of giving a reliable dose value when motorized scanning is used for the implantation. The maximum strain is $3 \%$ for sputtered films and $175 \%$ for implanted films.
Unsurprisingly, the sputtered sample exhibited the smallest strain range before loss of conductivity, which was limited to $3 \%$. At $1 \%$ strain, cracks started to appear perpendicularly to the direction of traction, and at $3 \%$ strain, the cracks became wide enough to completely interrupt the conducting path. In the case of implantation, the highly implanted samples could be stretched much less before ceasing to conduct than the lightly implanted ones. The maximal strain $s_{\max }$ was increased from $30 \%$ for an implantation dose of 800 pulses $\mathrm{cm}^{-2}$ to $175 \%$ for a dose of 400 pulses $\mathrm{cm}^{-2}$.

TEM images of membranes' cross-sections implanted at $5 \mathrm{keV}$ show that the cluster size increases with the dose, ${ }^{[16]}$ suggesting that a highly implanted gold layer exhibits characteristics that will tend toward those of a continuous metallic film. The dose must be controlled to obtain simultaneously very high maximum strain $(>50 \%)$ and a time-stable conductivity. The dose must be high enough to obtain a stable layer, and it must remain low enough to enable large strain capabilities. The strip implanted with 400 pulses $\mathrm{cm}^{-2}$ combines a low initial resistance of $398 \Omega$ (199 $\Omega$ per square) with an outstanding maximal strain before loss of conductivity of $175 \%$, at which point the strip's resistance is $1.1 \mathrm{M} \Omega$.

As seen in Figure 6, the higher the implanted dose, the higher the resistance increase rate versus strain is. This is not the only factor that reduces the maximal strain of the higher implanted samples. The maximal resistance of the strip, reached just before the layer becomes non-conductive, is much lower on the highdose samples. From $210 \Omega$ for the continuous sputtered film, it increases to $2.6 \mathrm{k} \Omega$ for the implantion at 800 pulses $\mathrm{cm}^{-2}$, and up to $1.1 \mathrm{M} \Omega$ for the lowest tested dose of 400 pulses $\mathrm{cm}^{-2}$. In the case of the sputtered layer, the limited increase in resistance before a massive loss of conductivity is explained by the cracks in the electrode: the film suddenly becomes non-conductive when the cracks grow large enough to break the conduction path. The situation is quite similar for the implanted samples: the high-dose electrodes have large clusters and a good conductivity, but when the clusters are separated and cease to touch each other, the conducting path is broken, even though the conductivity just before the path's interruption was still high. For the lower-dose samples that have many small clusters, the strain can be increased much more before all the conductive paths are broken, but as more and more paths break, the resistance increases to much higher values.

Similar tests were conducted with Pd implantation, with which stable low-resistance layers were also produced. A relation between dose and maximal strain was observed, similar to gold implantation, however, the maximal achievable strain was considerably reduced compared to $\mathrm{Au}$. The maximal strain varied between $10 \%$ and $33 \%$ depending on the dose.

Ageing and degradation of the resistance was studied for cyclic stretching between 0 and s' on a 500 pulses $\mathrm{cm}^{-2}$ Au-implanted sample, which is a dose that combines low surface resistance with large strain capabilities. The tested strip was cyclically stretched up to $s$ and the resistance measured every 100 cycles. More than 100000 cycles were conducted for $10 \%, 20 \%$, and $30 \%$ deformation, and the resistance at peak strain divided by the initial zero strain resistance is reported in Figure 7. The resistance at maximal strain increases with the number of cycles, and the increase rate depends on the strain level. For $10 \%$ strain, a 


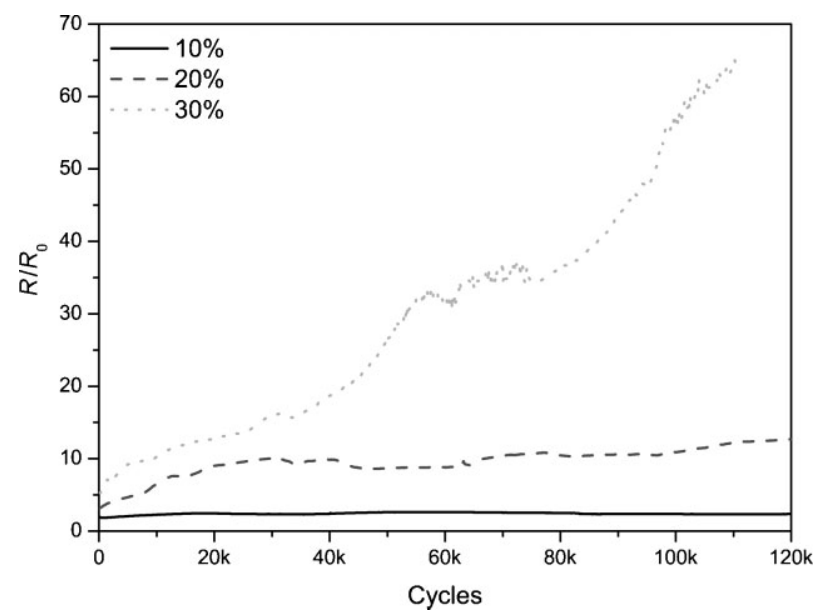

Figure 7. Degradation of the resistance of a 500 pulses $\mathrm{cm}^{-2} \mathrm{Au}$ implanted strip cyclically stretched to $10 \%, 20 \%$, and $30 \%$ for $100000+$ cycles. $R$ is the resistance of the stretched strip, and $R_{0}$ is the initial unstretched resistance.

small degradation is observed: the stretched strip's resistance exhibits only an increase of 32\% over 165000 cycles. Furthermore, the increase occurs during the first 10000 cycles, and the resistance remains stable afterwards. For the $20 \%$ strain, the degradation is more important, and an increase of $310 \%$ of the stretched value is observed over 123000 cycles. The degradation rate is higher for the first 30000 cycles, but a positive degradation rate is observed throughout the test. Finally, the test at 30\% shows an important degradation with a stretched resistance's relative increase of $1070 \%$, and a degradation rate which increases with the number of cycles. Table 1 summarizes the parameters and results of this test.

The degradation for three different strains presented in the previous paragraph were conducted on the same implanted strip, which therefore survived 399000 cycles while remaining conductive. Even though the strip's resistance underwent a large increase, it is still electrically usable for DEA applications, which do not require a very low resistance, as the response speed of actuators is generally mechanically rather than electrically limited. Similarly to what was observed for the maximal achievable strain, the degradation rate of a cyclically-stretched strip's resistance depends on the implanted metal dose. For highly implanted strips, the question is not about the increase of the resistance after a large number of cycles, but rather the number of cycles up to electrical failure of the layer: after 3000 cycles at $20 \%$ strain on a 800 pulses $\mathrm{cm}^{-2} \mathrm{Au}$-implanted strip, the resistance ceases to increase regularly, and a noisy chaotic

Table 1. Parameters and measured values for the ageing test on a 500 pulses $\mathrm{cm}^{-2} \mathrm{Au}$-implanted strip. $R_{0}$ is the initial unstreteched resistance; $R_{\max , 0}$ and $R_{\max , f}$ are the resistance at maximal strain at the beginning of the test and at the end.

\begin{tabular}{lllll}
\hline$s_{\max }$ & $R_{0}[\Omega]$ & $R_{\text {max }, 0} / R_{0}$ & cycles & $R_{\max , f} / R_{0}$ \\
\hline $10 \%$ & 354 & 1.96 & $165 \mathrm{k}$ & 2.60 \\
$20 \%$ & 391 & 3.15 & $123 \mathrm{k}$ & 12.9 \\
$30 \%$ & 1205 & 5.24 & $111 \mathrm{k}$ & 61.4 \\
\hline
\end{tabular}

resistance is exhibited due to irreversible damage to the microstructure. This is a drastically different behavior to the more lightly implanted sample, but part of the difference comes from the fact that a $20 \%$ strain represents $2 / 3$ of the maximal strain for the high-dose strip, while it is only $12 \%$ of the low-dose strip's maximal strain.

The strips implanted with doses of $400-500$ pulses $\mathrm{cm}^{-2}$ combine a low resistance (150-200 $\Omega$ per square) with a high maximum strain and a low degradation. The measurements show the existence of an optimal dose at which the best performance is obtained for the most important parameters: the samples which remain conductive at the largest strains are also the ones which have the longest lifetime in terms of cycles (slowest degradation). By comparison, the Pt electrodes of Urdaneta et al. exhibit low degradation for a Pt-salt concentration, which yields electrodes with small maximum strain, and faster degradation for conditions yielding electrodes with larger maximum strain. ${ }^{[13]}$

Stepped strain tests were performed on a Au-implanted strip with 500 pulses $\mathrm{m}^{-2}$ to observe the stability of the resistance when the sample is kept at constant strain for a few minutes. The strain was increased from 0 to $150 \%$ by steps of $10 \%$ and held for 10 minutes at each strain level (Fig. 8). The resistance remains very stable with time, even at large strain levels, which is an important difference when compared to what O'Brien observed on carbon-coated strips whose resistance exhibited noise as well as bumps. ${ }^{[18]}$

\section{Mechanical Properties}

For many flexible electrode application, the electrical resistance is the electrode's main parameter. However, obtaining low-resistance electrodes is not the only requirement for dielectric elastomer actuators. The conductive surfaces created on the elastomer must also have the smallest possible impact on the mechanical properties of the elastomer, in order to obtain large actuation strains. Quantifying the stiffening impact (i.e., the increase of the Young's modulus) caused by the implantation is consequently a necessary step in assessing the value of ion-implanted electrodes for DEAs. Additionally, precise determination of the mechanical (as well as

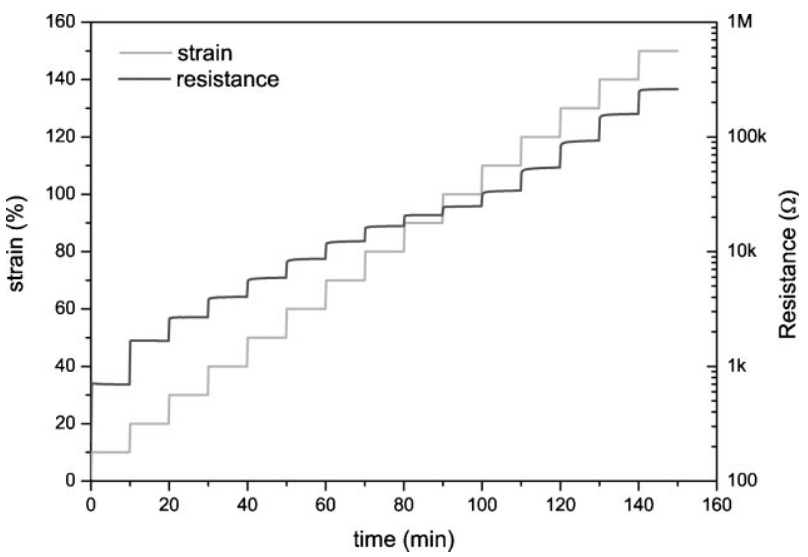

Figure 8. Resistance of an Au-implanted strip with 500 pulses $\mathrm{cm}^{-2}$ for different strains increased by $10 \%$ steps every 10 minutes, up to $150 \%$ strain. Resistance does not drift during the hold time. 
geometrical) properties of the PDMS membranes is necessary to obtain good predictions of our DEAs' behavior (displacement, mechanical work, etc.) by analytical models ${ }^{[15]}$

\subsection{Bulge Test Equations}

The bulge test is a measurement technique used to characterize the material properties of thin films: Young's modulus $(Y)$, residual stress $\left(\sigma_{0}\right)$ and Poisson ratio $(v)$ can be determined with such a test. A freestanding membrane of known geometry (shape, size and thickness) is submitted to a pressure $p$ on one of its sides. The deflection of the membrane's center $(z)$ is recorded, and material properties are extracted from the pressure-deflection curve, $p=f(z)$. This method has been successfully used to study the properties of silicon, ${ }^{[19]}$ silicon nitride, ${ }^{[20]}$ metallic ${ }^{[21,22]}$ and hard polymer ${ }^{[23,24]}$ thin films, as well as complex multilayer structures. ${ }^{[25]}$

Unlike the hard materials normally characterized by bulge tests, soft ( $Y \approx 1 \mathrm{MPa}$ ) PDMS membranes exhibit large deformations when submitted to a pressure. The bulge test equations found in the literature (see, e.g., Ref. [23]) are based on the assumption that the vertical deflection of the membrane during the bulge test is much smaller than the membrane's radius, which is not true for our membranes $(\varnothing 2-3 \mathrm{~mm}, 20-30-\mu \mathrm{m}$ thick) that can exhibit vertical deflection up to $20 \%$ of their radius when submitted to a pressure of $1 \mathrm{kPa}$. Additionally, because elastomers have a Poisson ratio of 0.5 , the vertical deformation leads to a thickness decrease of the membrane, which must also be taken into account. We have consequently derived a new set of equations applicable to our membranes.

The theoretical model which links the pressure on one side of the circular membrane to the deflection of its center is based on the assumption that the uniform pressure will deform the circular flat membrane into a spherical cap. In the case of thin membranes (thickness $t$ much smaller than the radius $r$ of the membrane), the relation between the biaxial stress $\sigma$ in the membrane and the applied pressure $p$ can be derived from the well-known equation for thin-walled spherical pressure vessels. ${ }^{[26]}$ The equilibrium of forces leads to:

$\sigma 2 \pi R t=p \pi R^{2} \Rightarrow p=\frac{2 \sigma t}{R}$

where $R$ and $t$ are respectively the radius and thickness of the vessel. In the case of a circular membrane of radius $r$ deformed into a spherical cap, the radius $R$ of the sphere can be calculated from the radius of the membrane, and the vertical deflection of its center $z$ :

$R^{2}=(R-z)^{2}+r^{2} \Rightarrow R=\frac{z^{2}+r^{2}}{2 z}$

The membrane's deformation into a spherical cap will increase its area, and due to the constant volume properties of elastomer this will lead to a decrease of the membrane's thickness:

$t=t_{0} \lambda_{z}=\frac{t_{0}}{\lambda_{a}}=t_{0} \frac{A_{0}}{A_{\text {cap }}}=t_{0} \frac{\pi r^{2}}{\pi\left(r^{2}+z^{2}\right)}=t_{0} \frac{r^{2}}{\left(r^{2}+z^{2}\right)}$, where $\lambda_{z}$ and $\lambda_{a}$ are respectively the thickness and area stretch ratios, and $t_{0}$ the initial membrane thickness.

The stress has two components: an elastic contribution due to the membrane's deformation, and the possible residual stress $\sigma_{0}$ induced by the fabrication process or by a voluntarily applied prestretch:

$\sigma=s \alpha Y^{*}+\sigma_{0}=s \alpha \frac{Y}{1-v}+\sigma_{0}$

where $Y^{*}$ is the biaxial Young's modulus, $Y$ the Young's modulus, $v$ is the Poisson coefficient, and $s$ is the strain. $\alpha$ is a parameter that depends on the Poisson coefficient, and which accounts for the fact that the stress state at the membrane's border is not equibiaxial due to the clamped boundary condition. Small and Nix have shown with numerical simulations that ${ }^{[26]}$

$\alpha \approx(1-0.24 v)$

The strain is calculated from the change of the arclength $\Delta l$ divided by the initial arclength, and the arclength $l$ is defined as:

$l=2 R \theta=2 \frac{z^{2}+r^{2}}{2 z} \arcsin \left(\frac{2 r z}{z^{2}+r^{2}}\right) \forall z \leq r$,

which can be simplified by replacing the arcsin function in the computation of the arclength (Eq. 6) by the first two terms of its power series:

$l \approx 2 r\left(1+\frac{2 z^{2}}{3 r^{2}}\right) \Rightarrow s \approx \frac{2 z^{2}}{3 r^{2}}$

where $s=(l-2 r) / 2 r$ is the strain. For a vertical displacement over diameter ratio $(z / 2 r)$ of 0.1 , the strain calculated with the complete formula is $2.65 \%$, whereas the above approximation gives a strain of $2.67 \%$, which is a very good approximation for a considerably simpler formulation. The pressure-deflection law is obtained by combining Equations 1-5 and 7:

$p=\frac{8(1-0.24 v) Y t_{0} z^{3}}{3(1-v)\left(r^{2}+z^{2}\right)^{2}}+\frac{4 \sigma_{0} t_{0} r^{2} z}{\left(r^{2}+z^{2}\right)^{2}}$,

The validity of the two models: elastomer model (Eq. 8) and standard bulge test model ${ }^{[23,26]}$ has been verified with finite element method (FEM) simulations. A model of a circular membrane with clamped boundary conditions was created with the FEM simulation package ANSYS and submitted to a uniform pressure on one of its sides. The membrane's mechanical and geometrical parameters were chosen to be representative values for our samples. The Young's modulus, stress, and Poisson ratio were respectively set to $1 \mathrm{MPa}, 30 \mathrm{kPa}$, and 0.5 . The thickness of the membrane was chosen to be $30 \mu \mathrm{m}$, and two different diameters were used: $2 \mathrm{~mm}$ and $3 \mathrm{~mm}$. The simulations were performed for pressures between 0 and $1200 \mathrm{~Pa}$, with $50 \mathrm{~Pa}$ pressure steps. Using the same parameters, the theoretical curve $p=f(z)$ was calculated with the three models (Fig. 9). The standard model diverges from the numerical data when the 


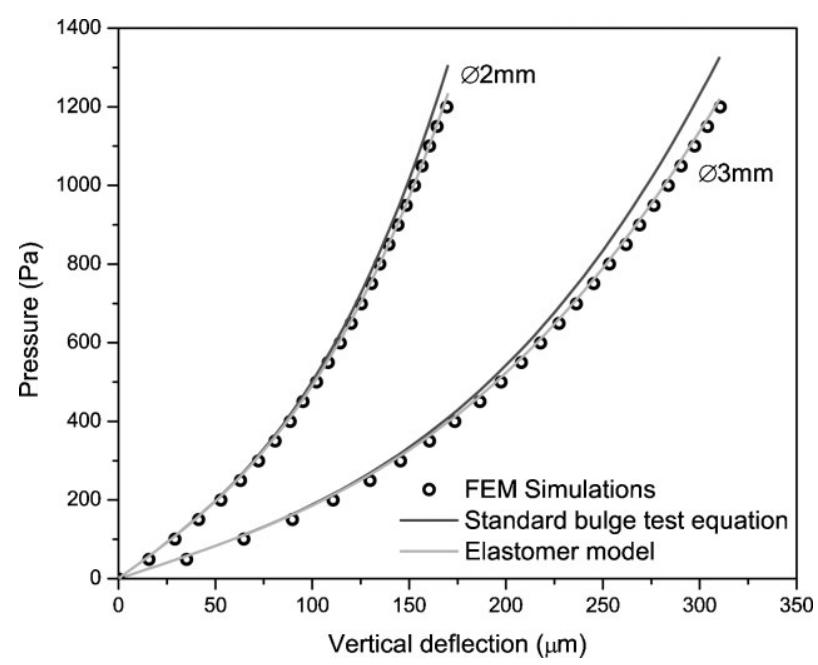

Figure 9. Comparison of the two bulge test theoretical models (standard, elastomer, and elastomer approximation) with FEM simulations for $Y=1$ $\mathrm{MPa}, \sigma_{0}=30 \mathrm{kPa}$, and $t_{0}=30 \mu \mathrm{m}$. When the vertical deflection becomes important relatively to the membrane's diameter, the standard model diverges from the simulated data.

vertical deflection becomes important relative to the membrane's diameter, because the assumptions on which it is based are no longer valid. This is particularly visible on the $\varnothing 3 \mathrm{~mm}$ membrane, for which the $z / 2 r$ ratio exceeds $10 \%$ at $1200 \mathrm{~Pa}$. The elastomer model provides a much better fit to the simulated data, thus proving its validity in the deflection and pressure region of interest.

\subsection{Bulge Test on Implanted Membranes}

Tension tests have shown that electrodes created by metal ion implantation can sustain large deformations while remaining conductive (cf. Sec. 4). However, another criterion must be taken into account in order to characterize the compliance of ionimplanted electrodes: the increase of Young's modulus induced by the addition of metallic particles into the PDMS. In a DEA, the thickness strain - as well as the area strain-is inversely proportional to the Young's modulus of the PDMS.$^{[9]}$ If the electrodes lead to an important stiffening of the actuator, its deformation capabilities for a given voltage will be reduced. A reasonable stiffening can be tolerated and compensated for by the application of a higher voltage, but if the stiffening effect becomes too important, the voltage that needs to be applied to compensate for the stiffening may exceed the breakdown field limit and destroy the device.

The relative increase of the Young's modulus $\left(\left(Y_{\text {impl }}-Y_{\text {PDMS }}\right) /\right.$ $Y_{\text {PDMS }}$ ) was calculated by measuring the Young's modulus of the membranes before implantation ( $Y_{\text {PDMS }}$ ), and after implantation with a given ion dose $\left(Y_{\text {impl }}\right)$ for different doses and metals. The results, obtained from bulge test measurements, are shown in Figure 10a. One can see that the stiffening due to $\mathrm{Au}$ and $\mathrm{Pd}$ implantations versus dose are very similar. Titanium implantation is shown to have the smallest impact on the stiffening of the PDMS membranes.
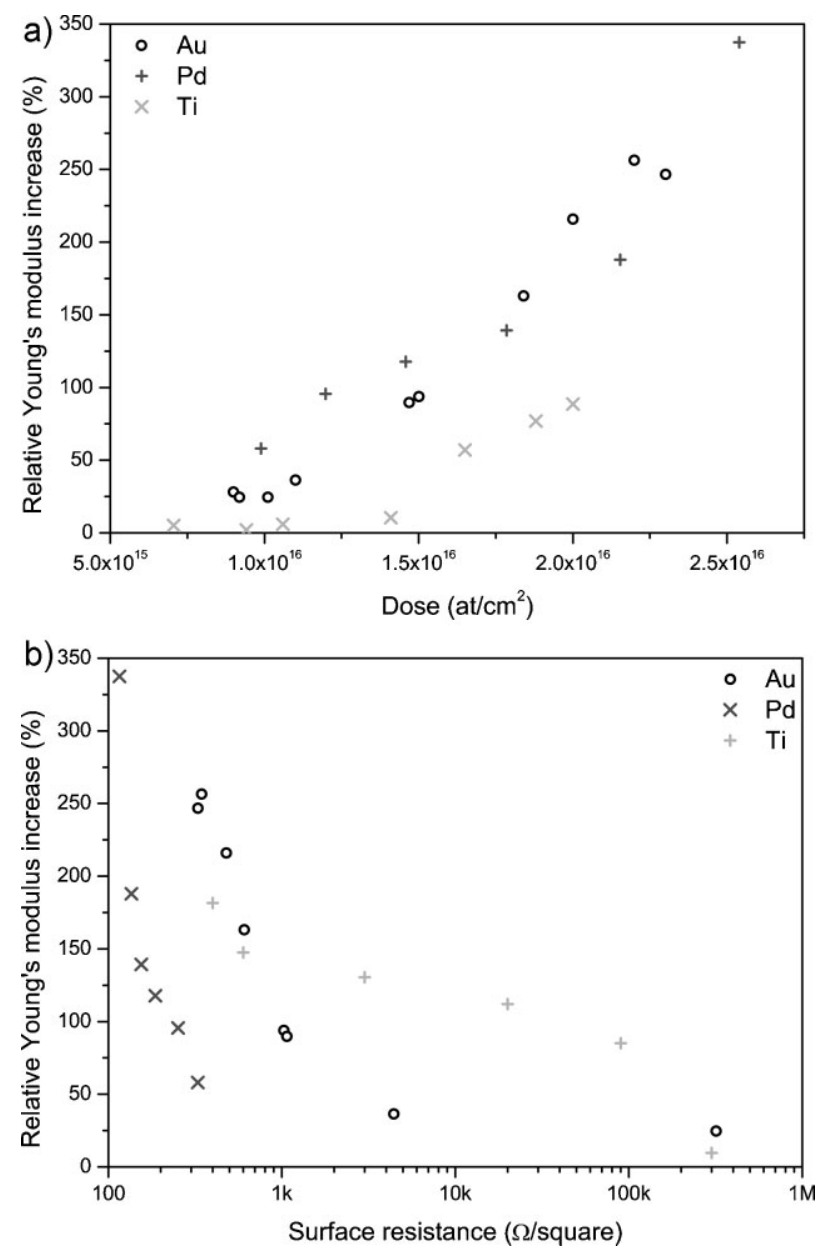

Figure 10. a) Stiffening of PDMS membranes for $\mathrm{Au}, \mathrm{Pd}$, and Ti implantations at different doses for a reference membrane with $Y_{\mathrm{PDMS}}=1 \mathrm{MPa}$ and $t_{0}=25 \mu \mathrm{m}$. b) Relative increase of the Young's modulus versus surface resistance obtained for the implantation of $\mathrm{Au}, \mathrm{Ti}$ and $\mathrm{Pd}$ on $25-\mu \mathrm{m}$-thick PDMS membranes with $Y_{\mathrm{PDMS}}=1 \mathrm{MPa}$.

Determining which metal is the most suitable to make compliant electrodes for DEAs can not be based solely on measurement of the stiffening caused by the implantation. If this measurement shows that Ti seems the most appropriate of the three tested metals, one has to keep in mind that titanium implantations failed to produce layers that had a time-stable resistance (cf. Sec. 3). It is therefore necessary to relate the implantation-induced variation of the mechanical parameters with the surface resistance obtained for the same implantation parameters. Plotting the stiffening as a function of surface resistance (Fig. 10b) clearly shows the general tradeoff between surface resistance and increase of Young's modulus, as well as the difference of properties obtained with the three implanted elements. Indeed, by looking solely at the stiffening versus dose measurements (Fig. 10a), one doesn't see any major difference between $\mathrm{Au}$ and Pd implantations. Adding the surface resistance information shows that Pd implantations allow to obtain a better surface resistance for an equal stiffening compared to $\mathrm{Au}$ implantations.

Minimizing the stiffening of the membrane should be the priority for DEAs, because having a very low resistance value is 
not necessary for electrostatic devices, as long as it is stable in time. Titanium, with its unstable surface resistivity, is therefore not useable. While the stiffening versus resistance plot indicates that $\mathrm{Pd}$ is the most appropriate of the three metals to make compliant electrodes, the fact that we were not able to obtain large strains while keeping electrical conduction for low-dose Pd implantations reduces the interest of this metal for DEAs. For other applications, for example electrodes for rollable displays, different conclusions would be reached as to best metal or dose.

\section{Dielectric Breakdown Field}

When developing electrodes for DEA applications, it is especially important to ensure that the method used does not reduce the maximal electric field that can be applied across the elastomer thickness before dielectric breakdown occurs. For example, carbon greases can diffuse into the PDMS, and techniques based on the inclusion of particles into the polymer (such as Pt salts, as described in ref. [13]) can have a negative impact the dielectric breakdown field. In the case of implantation, carbonization, chain scission and increase of surface roughness are likely to appear due to damage created by the dissipation of the incoming ion energy. Concerns can be raised on the influence of ion implantation on the dielectric breakdown of the PDMS, as it is possible that the implantation-induced damage could lead to a decrease of the dielectric strength of the PDMS.

We have carried out extensive dielectric breakdown field measurements on Au- and Pd-implanted PDMS, as well as on non-implanted PDMS, as previously reported. ${ }^{[27]}$ This study showed no difference in dielectric breakdown field between implanted and non-implanted PDMS. Furthermore, the obtained values $\left(>100 \mathrm{~V} \mathrm{\mu m}^{-1}\right.$ ) were much higher than the figures indicated in the PDMS datasheets, which are probably given with an important safety factor. The fact that ion implantation does not alter the dielectric strength of the PDMS is consistent with TEM micrographs, which showed a penetration of the metallic particles limited to $40-60 \mathrm{~nm}$, which is negligible compared to the thickness of our PDMS layers.

\section{Optical Transmission of Implanted Membranes}

Another interesting property of ion implanted electrodes is their optical transparency. Transmission measurements in the visible domain were conducted on Au- and Pd-implanted membranes at different doses (Fig. 11). Optical transmission is somewhat decreased after implantation due to light reflection from the metallic particles at the interface.

Unimplanted PDMS has a high optical transmission ( 90\% for $25-\mu \mathrm{m}$ membranes). Transmission decreases only weakly with dose, while remaining relatively high. Palladium implantation exhibits a monotonic relation between wavelength and implantation dose, with curves at different doses parallel to each other. At the highest tested dose, transmission remains above $56 \%$. For gold implantation, transmission depends on the wavelength and is lower (35\% for the highest dose) for red light, due to the very high reflection of IR by gold. The rather weak dependance on dose is because reflection-rather than
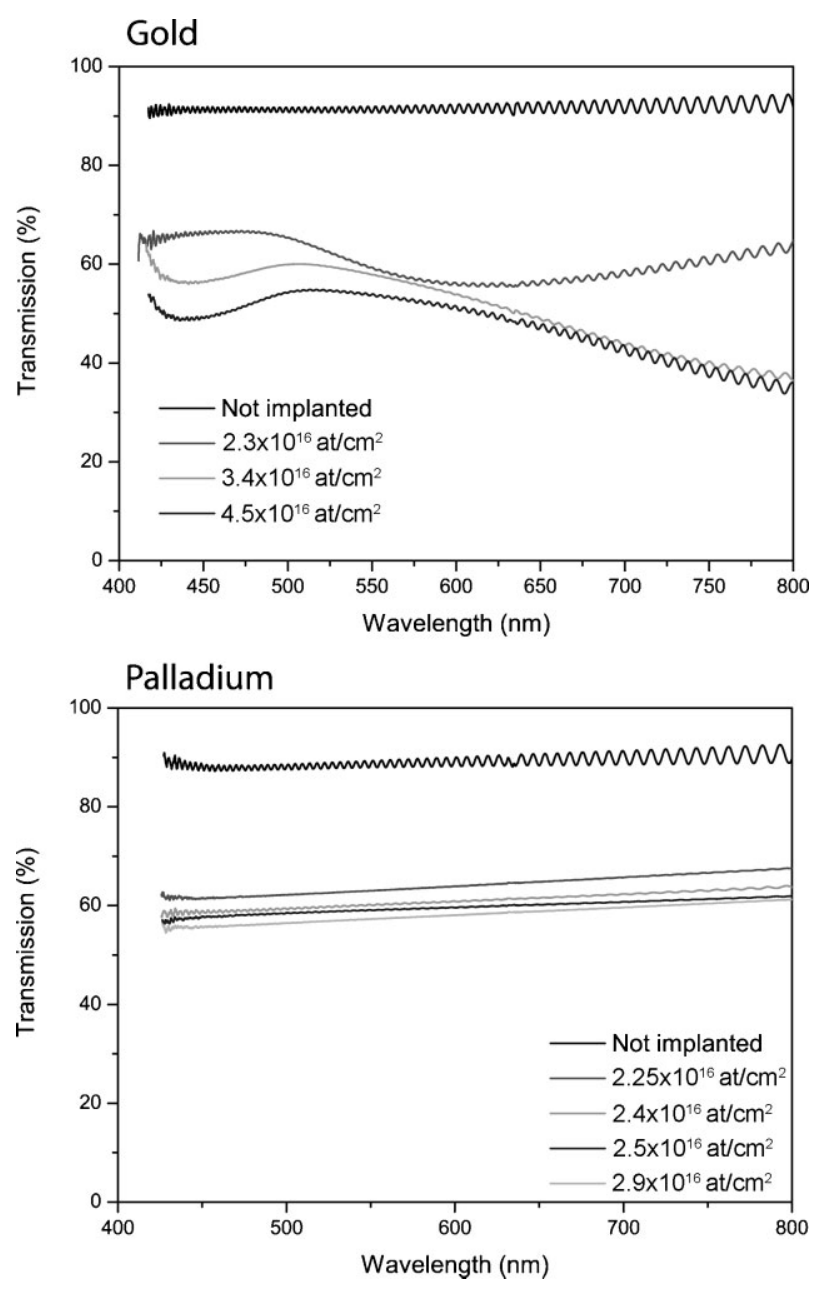

Figure 11. Optical transmission in the visible domain of PDMS layers $(t=25 \mu \mathrm{m})$ implanted with gold (top) and palladium (bottom). Oscillations are due to the Fabry-Perot effect.

absorption-dominates. The transmission is decreased due to reflection at the air/PDMS + metal interface, and the quantity of metal particles does not play a dominating role. For $\mathrm{Pd}$ implantation, one can expect $\sim 40 \%$ reflection at each interface.

Before implantation, the membrane is very smooth (surface roughness of pristine PDMS is below $2 \mathrm{~nm}$ root-mean-square, as measured by an atomic-force microscope) and interference at the membrane interfaces can be seen in the measurements. This is less visible on the implanted samples, particularly those with Pd, due to a surface roughness increase caused by the implantation process. These relatively good transmission properties are unique to implanted electrodes and cannot be achieved with thin-film or most of the carbon-based electrodes. This opens up a broad new field of applications for DEAs: those for which optical transmission is desirable.

We have also tested the resistance of implanted PDMS membranes to high-energy laser irradiation by submitting some samples to a pulsed IR laser beam. 1000 pulses of $10 \mathrm{~ns}$ duration with energy densities between 0.75 and $3.5 \mathrm{~mJ} \mathrm{m^{-2 }}$ pulse $^{-1}$ were sent through the membranes at rates varying between 5 and $20 \mathrm{~Hz}$. The implanted PDMS membranes were undamaged by those tests. 


\section{Conclusions}

We have shown that low-energy metal ion implantation is a highly effective fabrication technique to create compliant electrodes on elastomers. These patterned metal electrodes combine high (up to $170 \%$ ) maximal strain before loss of electrical conduction, a time-stable resistance (less than 1\% change over more than 8 months) and a low stiffening impact on the elastomer. Those three criteria are essential for fabricating EAP microactuators.

The three tested metals (gold, palladium, and titanium) exhibit very different behavior in respect of these three criteria. Gold is the tested metal that best fulfills the requirements of compliant electrodes for EAP applications. When a low implantation dosebut high enough to obtain a stable surface resistance value-is used, the resulting electrode can be stretched to strains above $170 \%$ and experiences an impact of $50-100 \%$ on the Young's modulus of the elastomer membrane, and can sustain over 100000 cycles at $30 \%$ strain. The same implantation conditions allow simultaneously obtaining i) high maximum strain without loss of conductivity, ii) large number of cycles at high strain, and iii) a minimal increase in the Young's modulus. This technique thus avoids any tradeoffs between these important parameters.

Because they remain conductive at high strain and can sustain large numbers of cycles without electrical or mechanical failure, metal-ion-implanted electrodes represent a promising technology to make easily patternable electrodes for electroactive polymer devices. The field of application of this technique goes however well beyond DEA-related devices, as there are many other applications, such as flexible electronics and biomedical devices, stretchable and rollable displays, which would benefit from these reliable electrodes.

\section{Experimental}

Polydimethylsiloxane: Two different commercial PDMS were used: Sylgard 186 from Dow Corning and Nusil CF19-2186 from Nusil. The very high viscosity of the two PDMS was lowered by adding isooctane with a weight ratio PDMS:Isooctane of 10:9 for Sylgard 186, and 10:6 for Nusil.

Suspended Membranes: Suspended PDMS membranes were fabricated by spin-coating a 20-30- $\mu \mathrm{m}$-thick PDMS layer on a flexible polyvinylidene chloride (PVDC) sheet fixed on a rigid frame and preliminarily coated with a sacrificial photoresist layer. Circular through-holes were patterned on a silicon wafer, either by laser drilling or by plasma etching. The cured PDMS layer was then bonded on the patterned Si by treatment in an oxygen plasma. Finally, the PVDC transfer substrate was removed by dissolution of the sacrificial layer in acetone.

Implantation: Metal ion implantation was conducted in a FCVA implanter based on an RHK Technology ARC 20 pulsed plasma arc source.

Gold Sputtering: Gold sputtering, both to make electrical contacts for the resistance measurement chips and to fabricated the PDMS strip with a sputtered electrodes, were made in a Balzers SCD-030 bench top sputtering system for $3^{\prime}$ at $60 \mathrm{~mA}$, leading to a thickness of $\sim 25 \mathrm{~nm}$.

Bulge Test Measurements: The pressure was applied by a motorized syringe pump and a $10 \mathrm{~cm}^{3}$ syringe through a $300 \mathrm{~cm}^{3}$ buffer volume, which allows for application of small pressure steps $(<1 \mathrm{~Pa})$ per increment of the stepper motor. The applied pressure was measured by a barometric pressure sensor MS5537 from Intersema with a measurement range between 0 and $3 \mathrm{kPa}$ and a resolution of $1 \mathrm{~Pa}$. The measurement of the membrane deflection was made with a white light interferometer Wyko NT1100 from Veeco.

\section{Acknowledgements}

This project has been funded by the Swiss National Science Foundation, grant \#200020-120164 and by the EPFL. The authors wish to thank Dr. Valentin Mitev, CSEM, for the high energy laser irradiations, as well as Dr. Massoud Dadras, CSEM, for access to the sputtering machine.

Received: August 18, 2008

Published online: December 18, 2008

[1] S. Ashley, Sci. Am. 2003, 289, 52.

[2] S. Takamatsu, K. Matsumoto, I. Shimoyama, in Proc. IEEE 21st Int. Conf. on Micro Electro Mechanical Systems MEMS 2008 2008, pp. 140-143.

[3] K. McGoldrick, in Proc. of the 36th European Solid-State Device Research Conf. 2006, pp. 1-2.

[4] D. H. Kim, J. H. Ahn, M. C. Won, H. S. Kim, T. H. Kim, J. Song, Y. Y. Huang, Z. Liu, C. Lu, J. A. Rogers, Science 2008, 320, 507.

[5] F. Carpi, P. Chiarelli, A. Mazzoldi, D. De Rossi, Sens. Actuators A 2003, 107 , 85.

[6] M. Aschwanden, A. Stemmer, Proc. SPIE 2007, 6524, 65241N-10.

[7] F. Carpi, C. Salaris, D. D. Rossi, Smart Mater. Struct. 2007, 16, S300.

[8] M. Gonzalez, F. Axisa, M. V. Bulcke, D. Brosteaux, B. Vandevelde, J. Vanfleteren, Microelectron. Reliab. 2008, 48, 825.

[9] R. Pelrine, R. Kornbluh, J. Joseph, R. Heydt, Q. Pei, S. Chiba, Mater. Sci. Eng. C 2000, 11, 89 .

[10] S. P. Lacour, J. Jones, Z. Suo, S. A. W. S. Wagner, IEEE Electron Device Lett. 2004, 25, 179.

[11] A. Pimpin, Y. Suzuki, N. Kasagi, J. Microelectromech. Syst. 2007, 16, 753.

[12] E. Seker, M. Reed, M. Utz, M. R. Begley, Appl. Phys. Lett. 2008, 92, 1541013.

[13] M. G. Urdaneta, R. Delille, E. Smela, Adv. Mater. 2007, 19, 2629

[14] S. Rosset, M. Niklaus, P. Dubois, H. R. Shea, in Proc. IEEE 21st Int. Conf. on Micro Electro Mechanical Systems MEMS 2008 2008, p. 503.

[15] S. Rosset, M. Niklaus, P. Dubois, H. R. Shea, Sens. Actuators A 2008, 144, 185.

[16] M. Niklaus, S. Rosset, M. Dadras, P. Dubois, H. Shea, Scr. Mater. 2008, 59 , 893.

[17] I. G. Brown, Surf. Coat. Technol. 2001, 136, 16.

[18] B. O'Brien, J. Thode, I. Anderson, E. Calius, E. Haemmerle, S. Xie, Proc. SPIE 2007, 6524, 652415-11.

[19] E. Bonnotte, P. Delobelle, L. Bornier, B. Trolard, G. Tribillon, J. Mater. Res. 1997, 12, 2234.

[20] J. J. Vlassak, W. D. Nix, J. Mater. Res. 1992, 7, 3242.

[21] A. J. Kalkman, A. H. Verbruggen, G. C. A. M. Janssen, Rev. Sci. Instrum. 2003, 74, 1383.

[22] V. Paviot, J. Vlassak, W. Nix, Mater. Res. Soc. Symp. Proc. 1995, 356, 579.

[23] B. E. Alaca, J. C. Selby, M. T. A. Saif, H. Sehitoglu, Rev. Sci. Instrum. 2002, 73, 2963.

[24] C. Poilane, P. Delobelle, L. Bornier, P. Mounaix, X. Melique, D. Lippens, Mater. Sci. Eng. A 1999, 262, 101.

[25] J. D. Hall, N. E. Apperson, B. T. Crozier, C. Xu, R. F. Richards, D. F. Bahr, C. D. Richards, Rev. Sci. Instrum. 2002, 73, 2067.

[26] M. Small, W. D. Nix, J. Mater. Res. 1992, 7, 1553.

[27] S. Rosset, M. Niklaus, V. Stojanov, A. Felber, P. Dubois, H. R. Shea, Proc. SPIE-Int. 2008, 6927, 69270W-10. 